\title{
Application of Composite Stone Simply Supported on Four Sides in Profiled Large Slab Stone Curtain Wall
}

\author{
Fenghui Li, Chao Xie, Zhiyan Che \\ China Construction Shenzhen Decoration Co Ltd, China Construction Engineering Corporation, Wuhan, China \\ Email address: \\ 41690781@qq.com (Fenghui Li),317162981@qq.com (Chao Xie),7959025@qq.com (Zhiyan Che)
}

To cite this article:

Fenghui Li, Chao Xie, Zhiyan Che. Application of Composite Stone Simply Supported on Four Sides in Profiled Large Slab Stone Curtain Wall. Engineering and Applied Sciences. Vol. 3, No. 5, 2018, pp. 121-128. doi: 10.11648/j.eas.20180305.11

Received: September 7, 2018; Accepted: October 25, 2018; Published: November 26, 2018

\begin{abstract}
With the development of curtain wall technology, along with the demand of artistic expression of architecture of curtain wall in China, some complex three-dimensional space shapes are increasingly used to the exterior of building. Among them, the construction of complex architecture formed by stone curtain wall system is extremely difficult. Currently, back-latching and hanging are the two most commonly used stone panel installation forms. The stone panel is a multi-point force bearing structure and the panel thickness of general stone curtain wall is about $30 \mathrm{~mm}$. In order to achieve architecture space shaping, on the one hand, stone panel thickness needs to be added at structural design. As the curtain wall dead weight is increased and the processing technology is more complex, the system safety and practicability can hardly be both considered. On the other hand, during installation on site, installation of stone panel for architectural shaping curtain wall is very difficult to control in terms of precision and the final installation quality and overall effect will be affected. In order to realize the architect's bold conceiving on the stone shaping of the commercial annex building of Chengdu Yintai Center, this project adopts a stone curtain wall system simply supported on four sides, greatly improving the mechanical properties of stone panel under the same condition. The lock block fastening method assures the installation precision. The construction tasks are completed highly efficiently with high quality, perfectly showing the unique visual effect of the stone curtain wall.
\end{abstract}

Keywords: Simply-Supported Hidden Frame Stone, Composite Stone, Large Slab Stone, Profiled SlaB Stone Curtain Wall, Three-Dimensional Modeling Analysis, Computer Space Positioning

\section{Introduction}

In pursuit of magnificence, energy saving, environmental protection and naturalness, natural stone is widely applied as interior and exterior wall facing material. In the 1930s, the stone curtain walls began to be used in high-rise buildings in the United States of America and made significant progress. In China, the wet bonding method was initially utilized to directly bond stone panels on exterior walls with cement mortar. The phenomenon of efflorescence was common on the surface of stone and the construction height was specified. Since the 1990s, the dry hanging method was introduced from abroad because it has great advantages in appearance, safety, durability and replaceability. Due to the update of process and technology, the dry hanging construction process gradually becomes the mainstream and the application scope of stone curtain walls has expanded rapidly.

Dry handing stone panels are connected in two forms [1]: later connection and back connection. After more than 30 years' application, the dry handing connecting structure of stone panels develop from the initial pin-type fixing system to the T-shaped fixing system, discontinuous edge rabbet and continuous edge rabbet, and later new fixing systems of back-latching, back-hanging and SE. The structure of dry hanging stone curtain wall is now developing towards the directions of being ecological, comfortable and intelligent. With the continuous improvement of dry fixing systems and the application of epoxy dry hanging glue, the height of the stone curtain wall has exceeded $200 \mathrm{~m}$. Practical experience shows that the discontinuous edge rabbet and back-latching stone panels with SE fixing systems are currently the mainstream dry hanging forms [2].

As the concept of green building becomes prevalent, the prefabricated members are attracting increasingly more attention. In terms of dry hanging stone systems, the unitized curtain wall and the composite curtain wall will become a 
development trend [3]. The unitized curtain wall is made of aluminum alloy with the basic curtain wall unit of a lateral grid at the storey height. Because most of the work in the system is carried out at the factory, the quality of processing and assembly can be guaranteed and the construction plan can be arranged in a coordinated manner. However, a single stone panel is generally around $1200 \mathrm{mmX} 3700 \mathrm{~mm}$, which means heavy weight, imposes high requirements on the lifting and installation during the construction process and leads to high cost [4]. In the composite stone curtain wall system, through reasonable structure and structural design, the stone panels are independent of each other and can be disassembled and installed at any time, thus making it easy to maintain the stone curtain wall. The stone curtain wall system can be safer and more reliable after changing the force bearing model of stone panels by reasonably arranging the number and position of connection points. The system is based on the basic unit with one lateral grid and one vertical grid. The panel size is about $1 \mathrm{~m}$. It is lightweight and convenient for installation, with the advantages of the unitized curtain wall. The sub-frame fabrication can be carried out concurrently with the keel installation on site. This may shorten the construction period, make it easier to produce in factories and control quality, and have good corrosion resistance [5].

According to the above studies, in order to overcome a series of problems, such as considerable difficulty in design and construction of the complex architectural form constituted by stone curtain wall system with unprepossessing appearance, based on the composite stone curtain wall, this project utilizes an aluminum alloy sub-frame that is bonded to four sides of the stone panel by using the epoxy resin $\mathrm{AB}$ glue. The exposed surface of the sub-frame applies the same type of fluorine carbon coating as that used by stone panels. The connecting piece is connected to the stone keel by stainless steel bolts. After the stone panel is mounted in place, aluminum alloy lock block and stainless steel bolts are employed to fasten the sub-frame and the connecting piece. The method is processing aluminum alloy sub-frame and stone panel into a whole. The connecting piece is installed on steel keel and fastened by aluminum alloy lock block and stainless steel bolts at the corresponding positions. The anti-corrosion washer is placed between the sub-frame and the connecting piece to achieve the connection of stone panel and steel keel, so that the stone panel forms a force bearing structure simply supported on four sides.

The stone curtain wall system with a hidden frame and simply supported on four sides can significantly improve mechanical properties of the stone panel under the same conditions, and the lock block fastening can ensure the installation precision. The visual effects can be adequately conveyed. During the construction process, the installation efficiency on site is greatly enhanced while the construction period is shortened accordingly.

The total station is utilized for data acquisition and three-dimensional modeling analysis. According to the analysis results, the curtain wall frame is accurately positioned and the panel is divided. The computer spatial positioning and construction actualities are integrated for scientific supervision of construction, thus considerably improving the installation precision. This method is applicable to the construction of curtain walls with irregular grids or profiled slab stone curtain walls in office buildings, exhibition venues, sports stadiums, airports and industrial factories. If the work schedule is very tight, this method can significantly enhance the quality of on-site installation and successfully complete the construction tasks assigned by the employer.

\section{Advantages of Composite Stone Simply Supported on Four Sides in Profiled Large Slab Stone Curtain Wall}

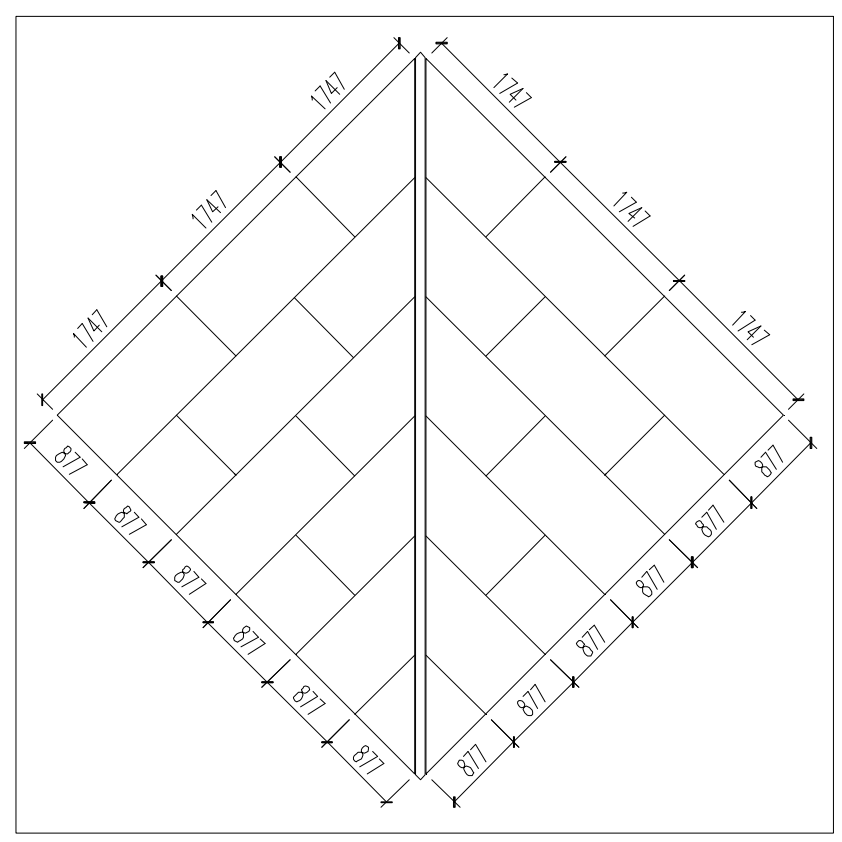

Figure 1. Stone panel grid of Commercial Annex Building of Chengdu Yintai Center.

The stone curtain wall of the commercial annex building of Chengdu Yintai Center is unique in shape and all the stones are installed obliquely by 45 degrees, and the largest single panel is $1.5 \mathrm{~m}^{2}$ (and the largest specification is $1747 \times 877 \mathrm{~mm}$ ).

This stone curtain wall is achieved through back-latching connecting method. Through verification, the stone thickness needs to reach $35 \mathrm{~mm}$ and requires 6-point back-latching support [6]. The single stone panel is about $140 \mathrm{~kg}$, so the installation is extremely difficult. In addition, it is also very difficult to carry out back-latching position-limit operation for obliquely installed stone.

In order to achieve the unique visual effect of such stone curtain wall in a perfect way, this project adopts a curtain wall system of composite stones simply supported on four sides. Specifically, an aluminum alloy sub-frame [7] is installed along the notched stone (to form an entirety using the angles combined from the aluminum alloy sub-frame). A perfect combination between the sub-frame and the stone is achieved by applying epoxy resin $\mathrm{AB}$ glue inside the notch [8], so that 
the stone and the frame can jointly bear the force. With polyurethane (PU) filled in the back of stone and calcium silicate board in place, the PU foaming generated via high-pressure equipment effectively integrates the stone, the sub-frame, PU and the calcium silicate board as a whole. The compounded stone shares force together with the sub-frame.
Validation shows that $25 \mathrm{~mm}$-thickness stone is strong enough to meet the force bearing requirement [9]. Stone thickness is reduced in an effective way by changing the six-point support of facing stone into simple support on four sides so that the weight of facing slab is reduced for the convenience of installation.

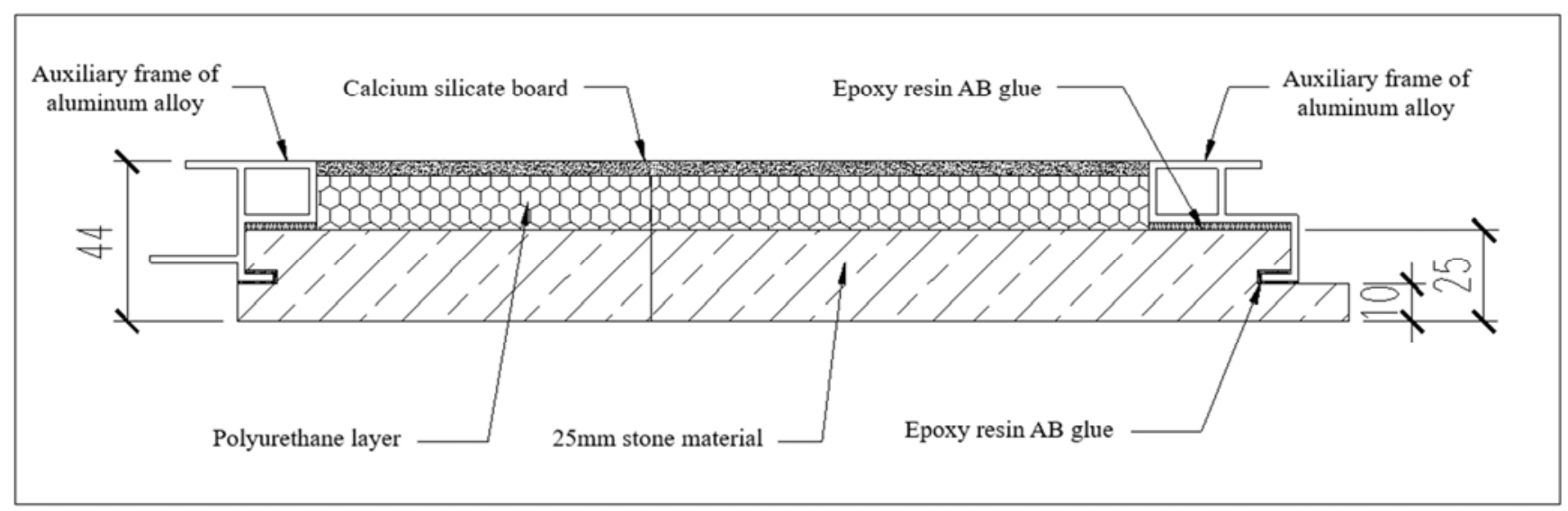

Figure 2. Sectional drawing of composite stone simply supported on four sides.

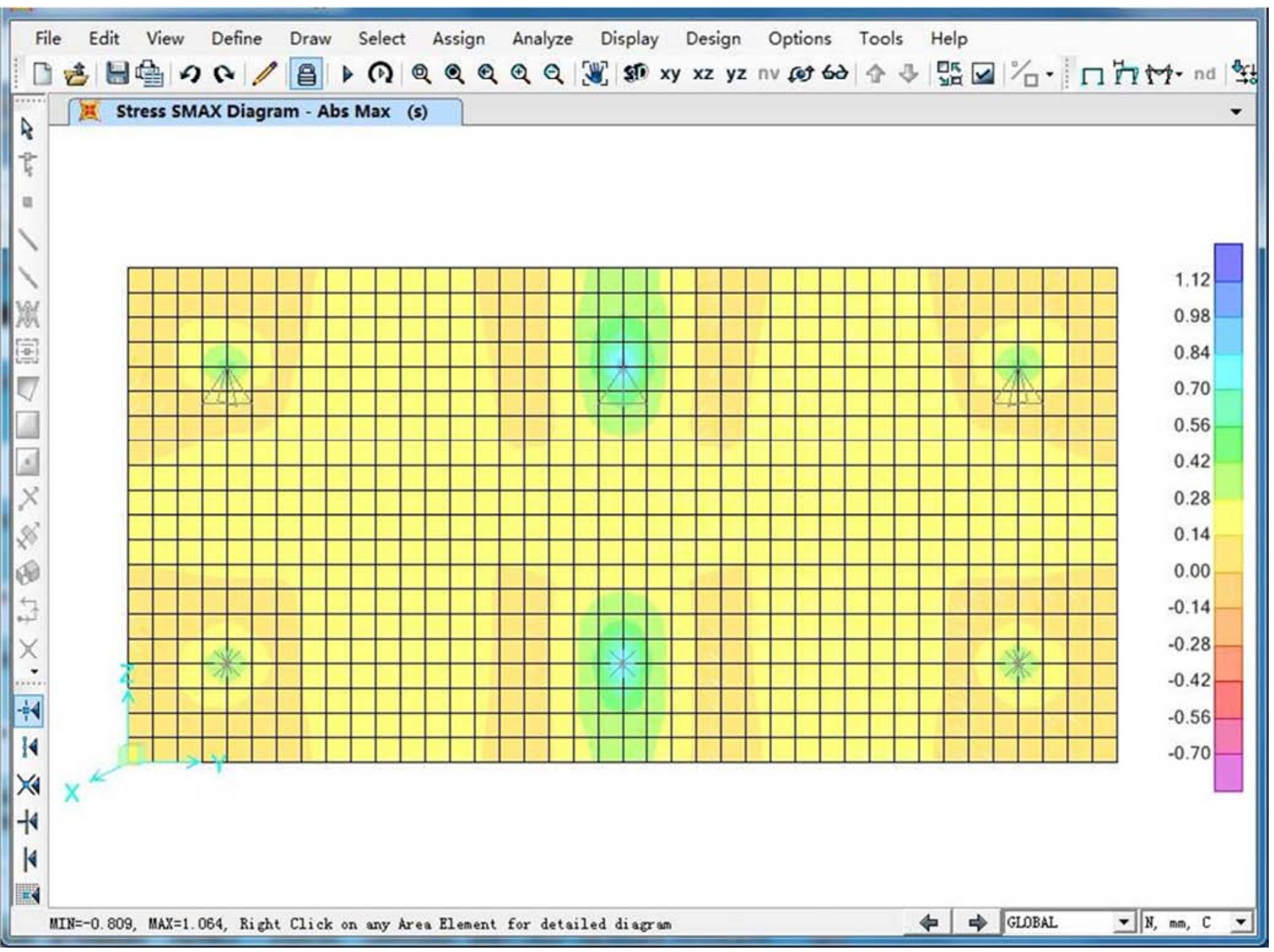

Figure 3. Analytical drawing of 35mm six-point support back-latching stone strength. 


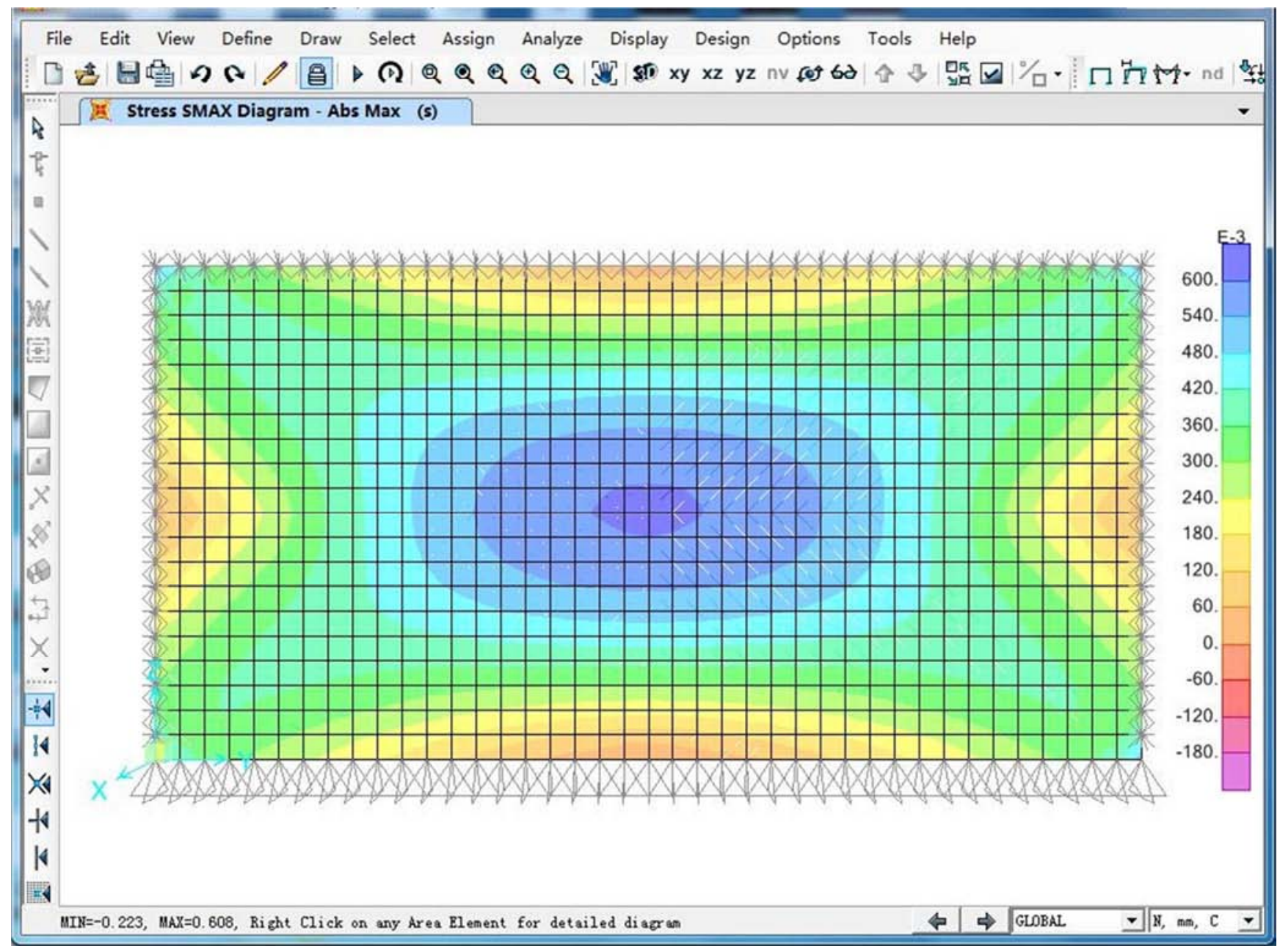

Figure 4. Analytical drawing of strength of $25 \mathrm{~mm}$ stone simply supported on four sides.

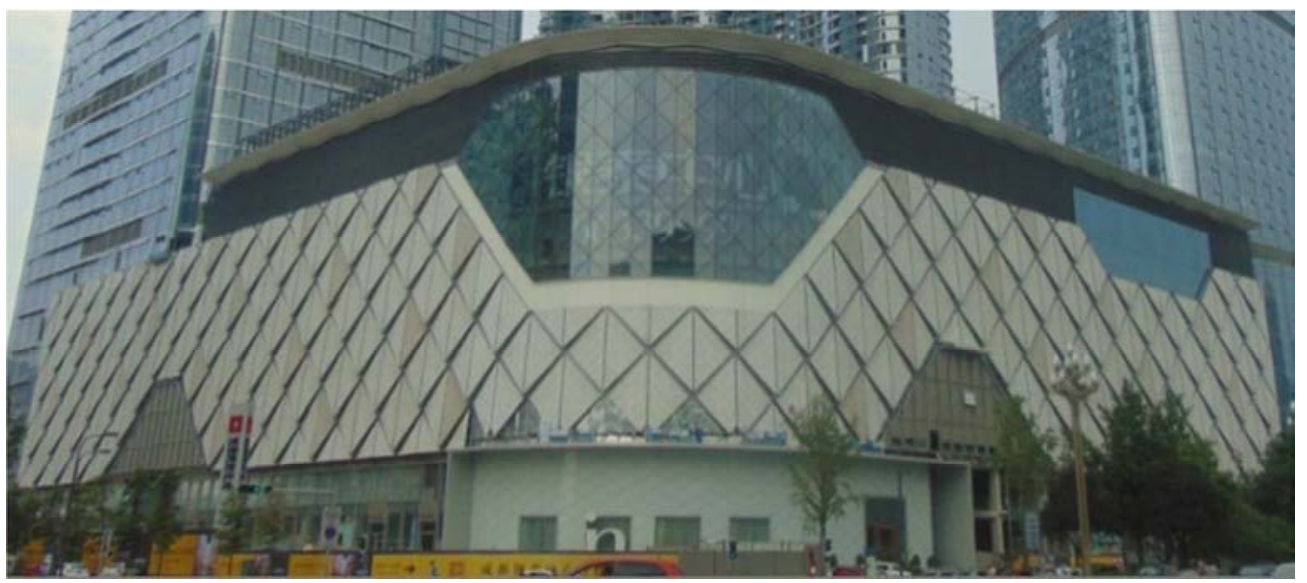

Figure 5. Overall effect of stone curtain wall of commercial annex building of Chengdu Yintai Center.

Through changing the force bearing pattern of stone, this project uses stones simply supported on four sides instead of common six-point support back-latching stone, greatly improving the mechanical performance of stone panel under the same condition [10]. In addition, the lock block fastening method ensures the installation precision. The construction tasks are completed efficiently with high quality, and the unique visual effect of stone curtain wall is perfectly achieved.

\section{Key points of Processing Composite Stone Simply Supported on Four Sides}

\subsection{Flowchart of Composite Stone Processing}

1) Stone material cutting: The stone material is cut on the surface along the side by a certain angle and size; stone 
material notching: The U-shaped groove is notched on the surface along the side of the stone material by a certain depth and width or by a certain angle.

2) Stone surface cleaning: The stains on the stone surface can be washed with neutral solvent and rinsed with water.

3) Stone surface protective treatment: A layer of flexible protective film is laid on the contact surface between the stone material and the work bench to prevent scratches on the surface of the stone material.

4) Installation of aluminum alloy sub-frame: The aluminum alloy sub-frame is adhered to the stone panel by applying the epoxy resin $\mathrm{AB}$ glue.

5) Stone material composition: The PU panel is fastened to the back of the stone panel and then the calcium silicate board is fixed to the back of the PU panel.
6) Stone material clearing: The glue or stain on the stone material surface is scraped off with a blade, washed with neutral solvent and rinsed with water. Scrubbing or scratching with a blade is not allowed. Only the solvent and water can be used for cleaning.

7) Accepted in the self-inspection. After being made in the factory, the composite stone material must be self-inspected and can be used for installation after passing the flatness acceptance.

8) Delivered to the construction site: During transportation of composite stone materials, the package of four corners should be contacted. It should be neatly placed and firmly bound with ropes, so as to prevent friction of materials due to bumps en route.

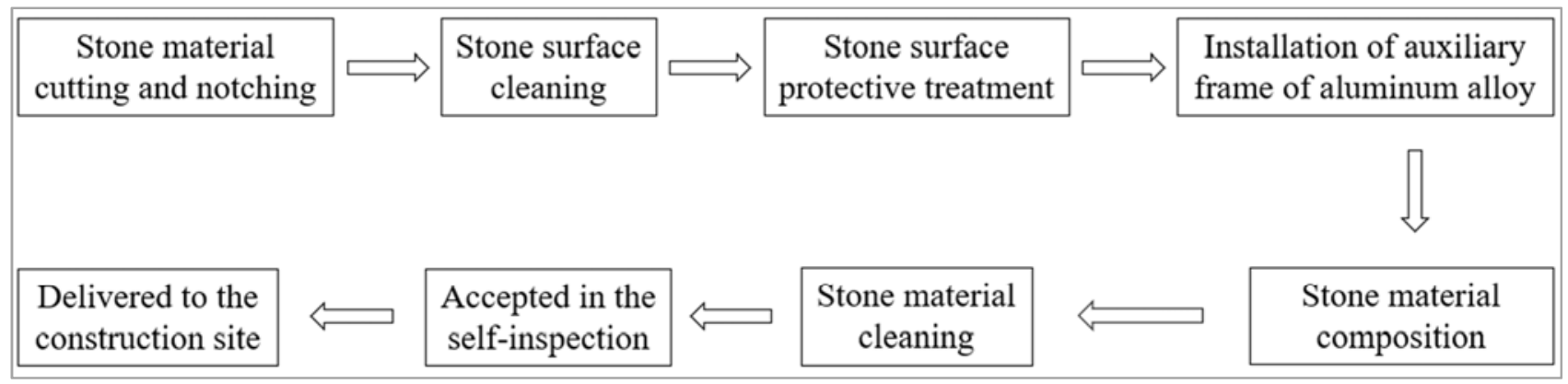

Figure 6. Flowchart of composite stone processing.

\subsection{Key Points of Quality Control}

1) Epoxy resin $\mathrm{AB}$ glue [11] needs to be applied inside the stone notch before installing aluminum alloy sub-frame. The sub-frame installation should be flat with tight jointing at gaps.

2) Gaps between aluminum alloy sub-frame and stone require the filling of epoxy resin $\mathrm{AB}$ glue.

3) Angle aluminum is used for connection between aluminum sub-frame to form a continuity of aluminum frame [12].

4) After stone composition, check if the stone edges are broken; stone with broken edges shall not be delivered to the construction site.

\subsection{Illustration of Composite Stone Processing}

The patterns are distributed according to the division of the diamond-shaped stone curtain wall. The stone panels are arranged and those with large color differences are eliminated. Then they are adhered to the four sides of stone panels by applying the epoxy resin AB glue. After the glue becomes dry, the fluorine carbon coating with a similar color with stone material is applied to the aluminum profiled sub-frame and hence the composition of aluminum alloy sub-frame and stone panel is completed.

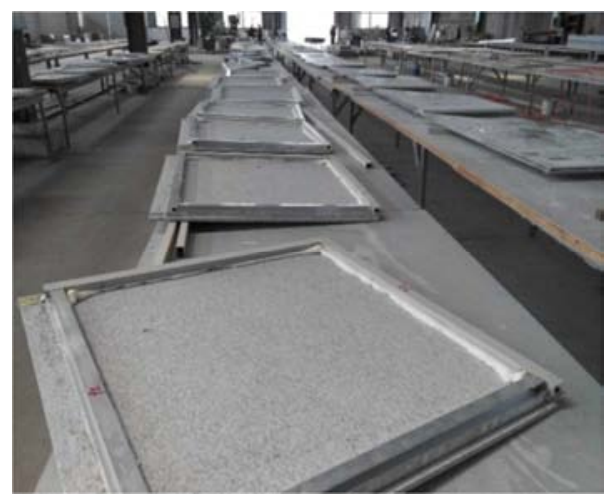

(a) Stone notching

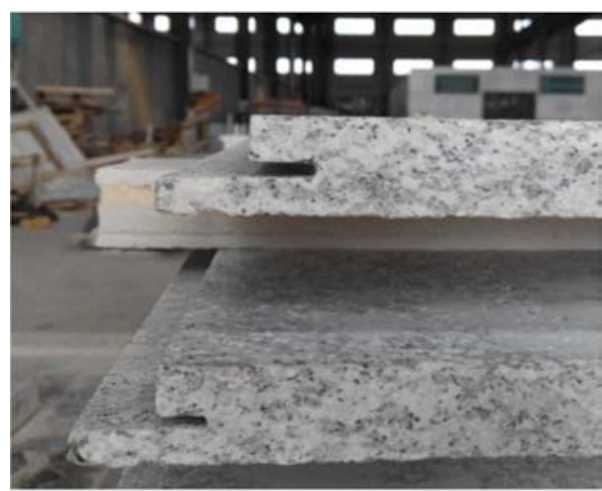

(b) Aluminum alloy frame and stone fixation 


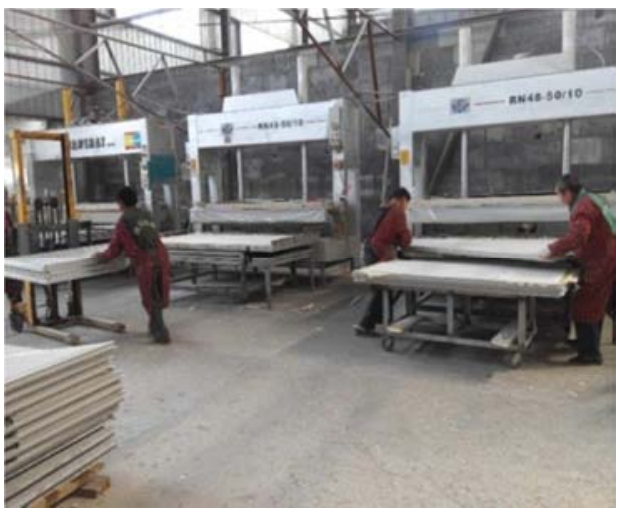

(c) Apply PU, place calcium silicate board

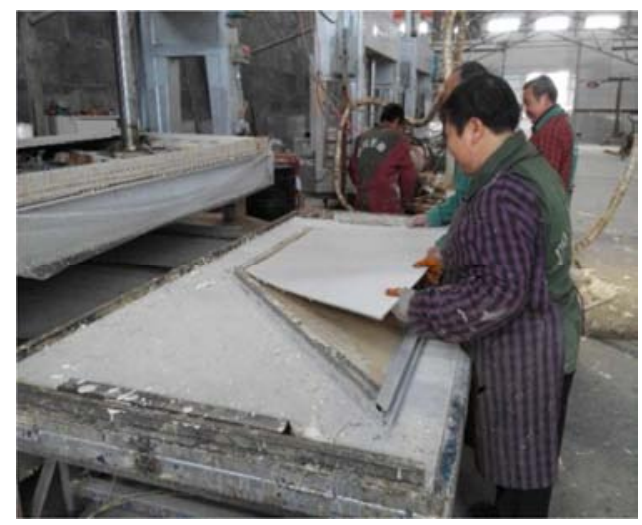

(d) Enter composition equipment for composition

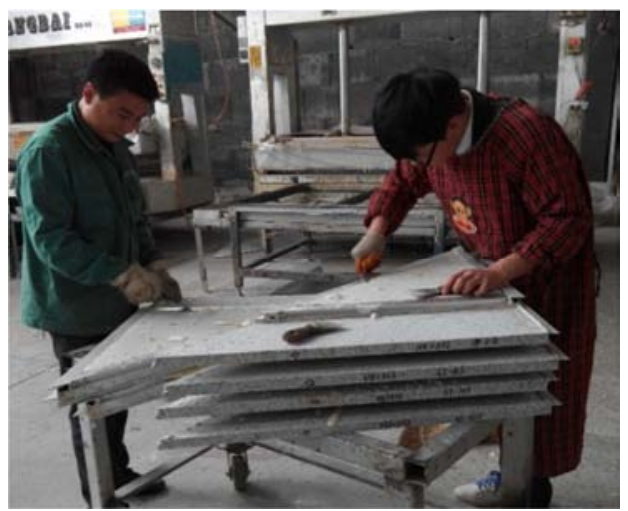

(e) Composition completed and packaging

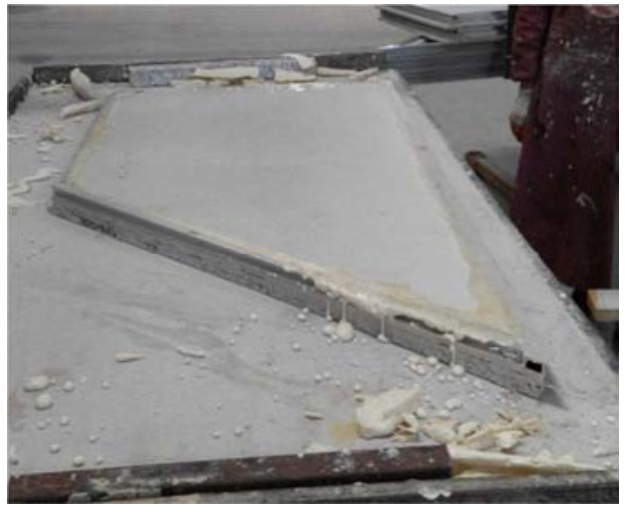

(f) Ready for delivery after cleaning

Figure 7. Illustration of composite stone processing.

\section{Field Installation of Composite Stone Simply Supported on Four Sides}

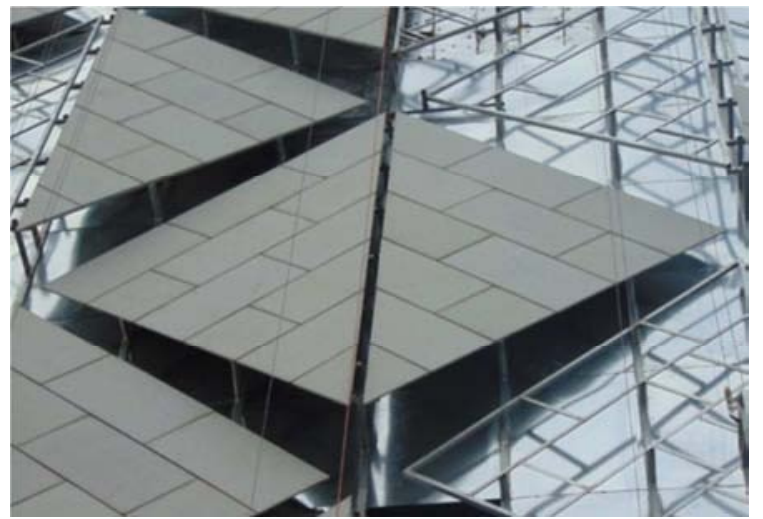

Figure 8. Picture of installing process of composite stone simply supported on four sides.

In this project, the stone curtain wall uses rhombus-shaped stones as the basic units. One rhombus unit contains two triangles. As the stones are obliquely installed by 45 degrees and the curtain wall sub-keel requires a lot of welding with difficult space positioning, in order to effectively solve the problem of stone curtain wall sub-keel installation, a custom-made overall lifting plan for sub-keel triangle steel frame is used in construction. That is to say, bed-jig is made from sub-keel steel triangle frame welding in the workshop, which not only provides convenience to precise positioning of each sub-keel, but also effectively controls welding deformation to ensure welding quality; In addition, it greatly improves processing and installation quality, so the construction period is effectively guaranteed [13].

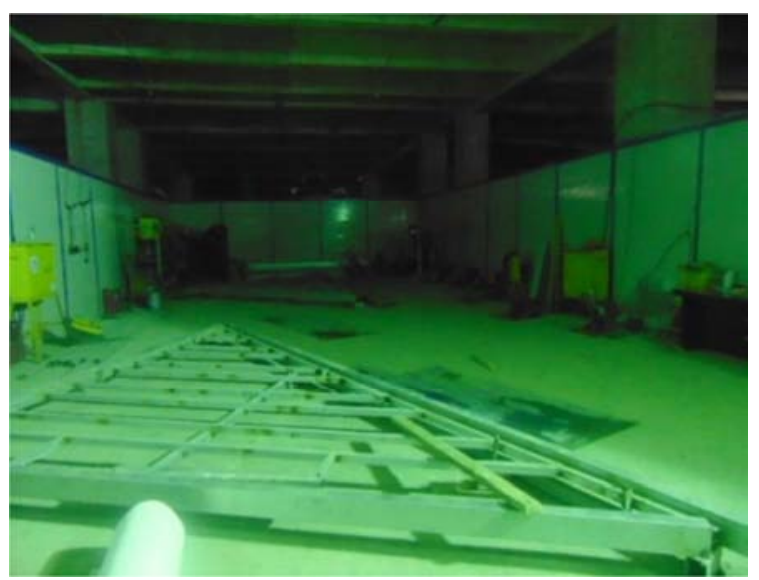

Figure 9. Making bed-jig from triangle frames.

Pursuant to the construction drawings and the re-measurement on site, the project department plots the keel processing drawing, welds the keel in the factory and delivers it to the project site for lifting and installation.

The advantages of lifting stone keel by sections include reduction of on-site processing time, better welding environment in the factory than on the site, assurance of keel welding quality and improvement of installation efficiency. 


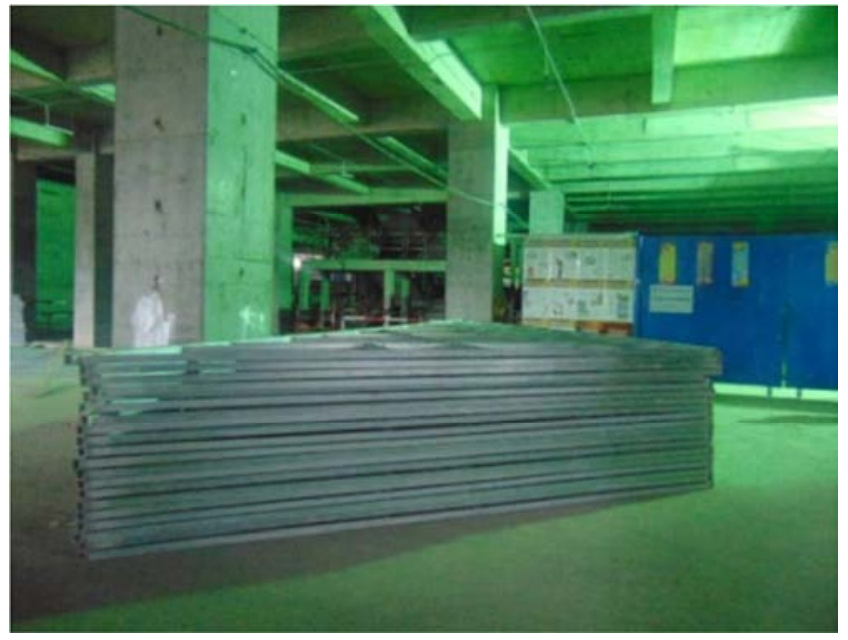

Figure 10. Triangle frame fabrication.

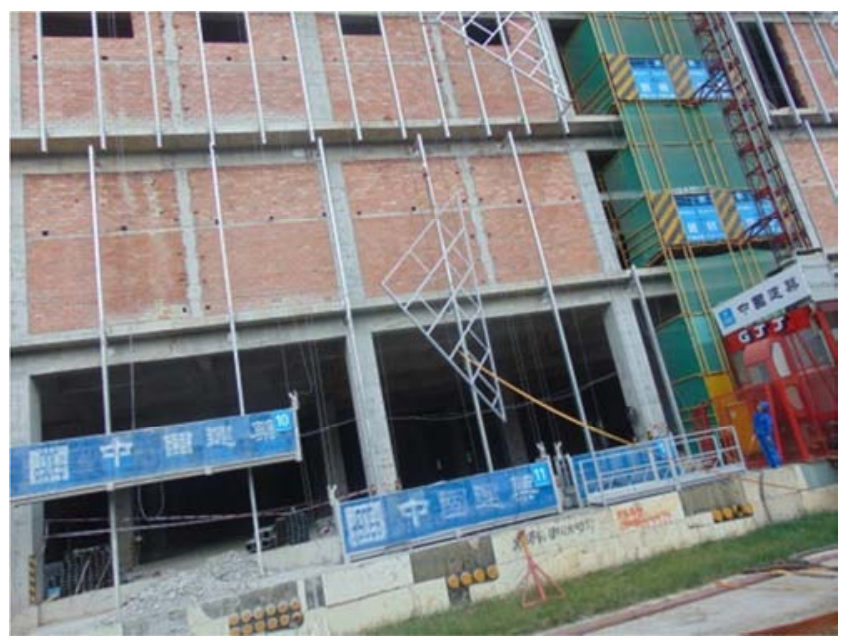

Figure 11. Overall lifting of triangle frame.

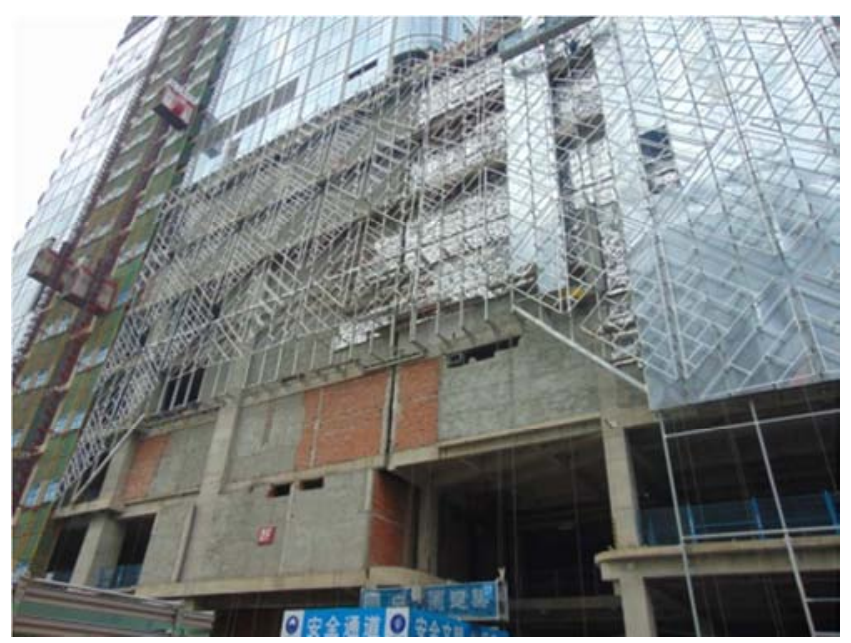

Figure 12. Picture of stone sub-keel triangle frame installation.

When the stone triangle frame is processed and made, the installation hole position of the stone connecting piece is processed by directly using setting out on bed-jig. After the installation of the upper wall of the stone steel triangle frame, the connecting piece is directly installed. When the connecting piece is installed, it is necessary to ensure that the finished surface of the stone connecting piece in the same triangle frame must be in the same finished surface. This is the key factor to determine whether the stone installation is smooth or not.

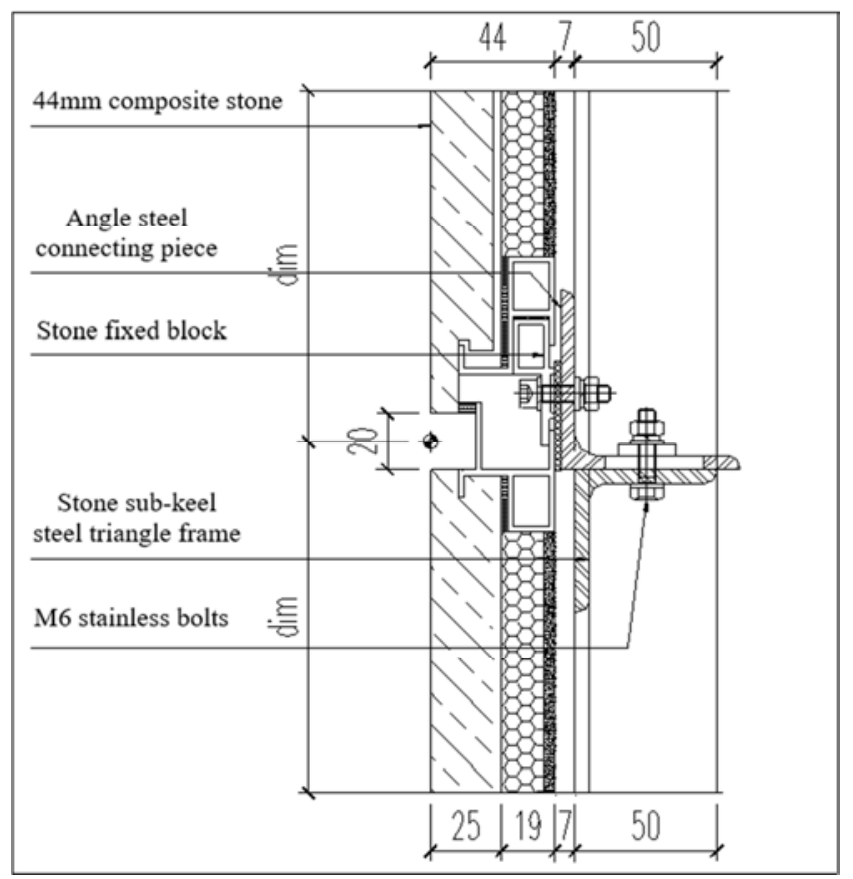

Figure 13. Standard installation node of composite stone simply supported on four sides.

Installation of the first stone panel: as this system is installed by means of directional mounting, the first stone needs to be prepared with aluminum alloy frame lock block before it is hung up for installation.

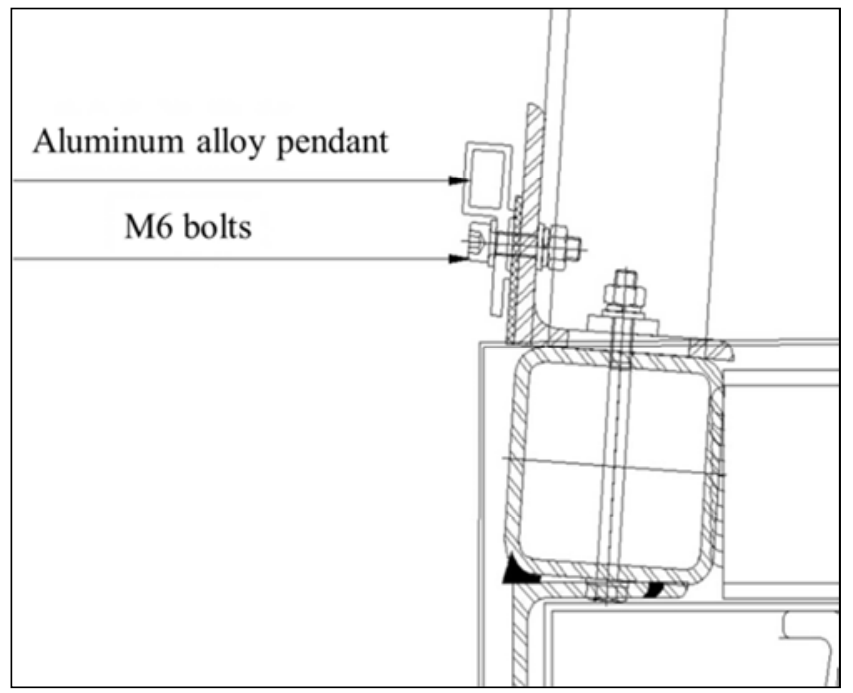

Figure 14. Mounting installation of aluminum alloy.

The stone panel is installed through man-basket. After pre-positioning, it is positioned by slightly adjusting the chain block. After positioning, it is fixed and fastened using lock block. The bottom-up order is followed for installing each triangle frame stone. 


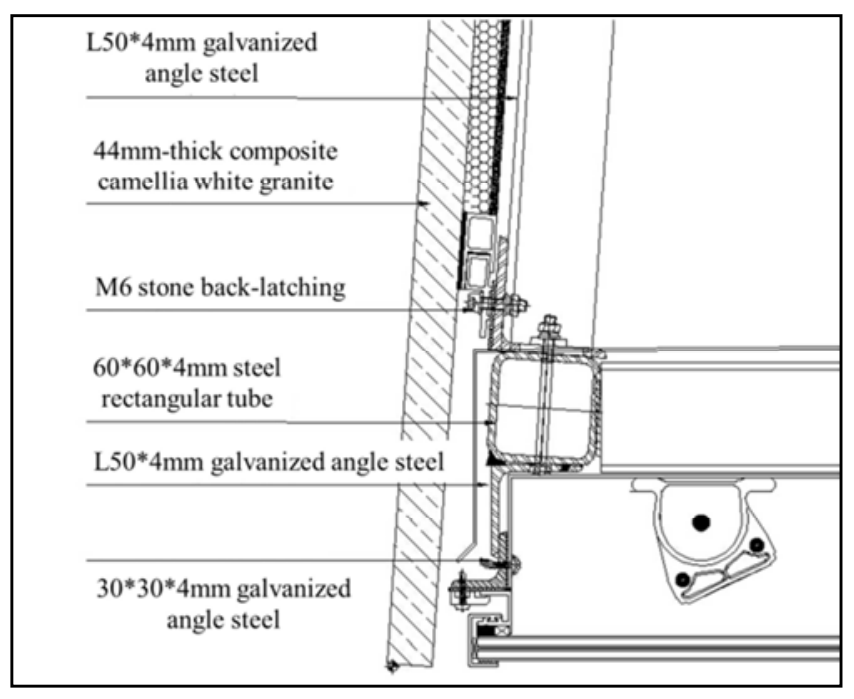

Figure 15. Installation of the first stone.

For each stone installation, from installation to completion, the quality control is carried out throughout the whole process, and the acceptance is also carried out intermittently in the whole process. The acceptance contents include: if the stone panel has defect, if the size complies with design requirement, if there is any staggering face, if the stone joint complies with the design requirement, if the stone connecting pieces within the same triangle frame are in the same plane, if the number of stone blocks meets the design requirements, and if the gap between stones meets the design requirements. The acceptance record and the upper lock block belong to the scope of the concealed project, and all kinds of information should be prepared according to the relevant provisions of the concealed project.

The glue or stain on the stone material surface is scraped off with a blade, washed with neutral solvent and rinsed with water. Scrubbing or scratching with a blade is not allowed. Only the solvent and water can be used for cleaning.

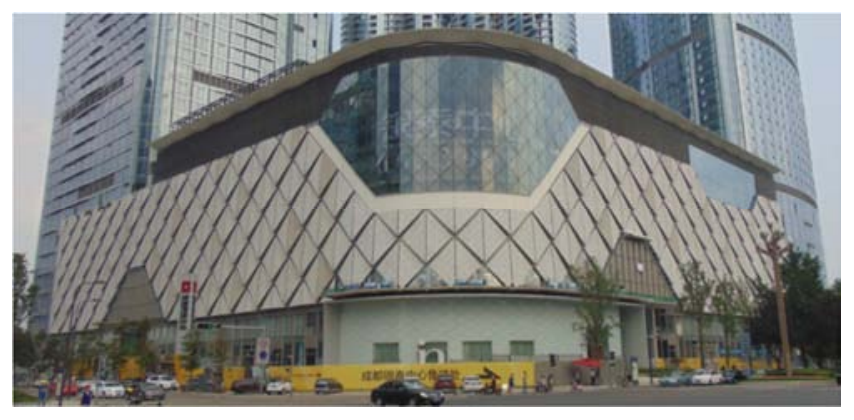

Figure 16. Picture of stone curtain wall of Chengdu Yintai Center.

\section{Conclusion}

Currently, the installation of stone curtain wall of commercial annex building of Chengdu Yintai Center is already completed. This stone curtain wall perfectly shows the architect's conceiving of artistic effect of elegance, modesty and liveliness, casting beautiful reflection with the high-end positioning of Yintai commercial complex. The project is unanimously praised by the public. Under the impact of the social themes of environmental protection and energy saving at present, it has achieved good social benefit in terms of reducing construction period and improving installation precision.

It is believed that the composite stone simply supported on four sides will win more markets and create more elite projects for the society with its good mechanical property, high installation precision, fast construction speed and other advantages.

\section{References}

[1] Fan Li. Study on the advantage of dry hanging method for back bolt type stone curtain wall [D]. Chang'an University, 2011.

[2] Bing Zeng. Application research progress of dry hanging stone curtain wall system [J]. Adhesion, 2016(10):53-56.

[3] Xuwang Cui, Research on construction technology of ultra-thin stone thermal insulation integrated exterior wall decorative panels [J]. Building Construction, 2015(8):951-952.

[4] Lei Zhang. Installation technology of complex special-shaped stone curtain wall in a project [J]. Construction Technology, 2013, 42(14):43-46.

[5] Xiajun Zhang. Construction technology of special-shaped curved stone curtain wall in YinChuan Railway Station [J]. Construction Technology, 2011, 40(22):24-26.

[6] GB/T21086-2007, Building curtain wall [S].

[7] GB/T3880.2-2012, General industrial aluminum and aluminum alloy plates second parts: mechanical properties [S].

[8] JC/T887-2001, Epoxy adhesive for dry hanging stone curtain wall $[\mathrm{S}]$.

[9] GB50009-2012, Load code for the design of building structures [S].

[10] GB/T18250-2000, Detection method for in-plane deformation performance of building curtain wall [S].

[11] GB/T14682-2006, Terminology of building sealed materials [S].

[12] JGJ/T216-2010, Construction specification for aluminum alloy structural engineering $[\mathrm{S}]$.

[13] JG/T216-2008, Small unit building curtain wall [S].

[14] JGJ133-2001, Technical specification of metal and stone curtain wall engineering $[\mathrm{S}]$.

[15] GB50210-2018, Quality acceptance standard for Building Decoration Engineering [S]. 